\title{
EDITORIAL
}

For reprint orders, please contact: reprints@futuremedicine.com

\section{Multiplexed point-of-care assays for HIV and co-infections for resource constrained settings: a perspective}

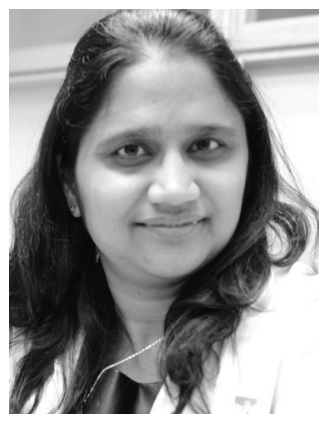

Nitika Pant Pai*

\author{
"The impact of multiplexing is very \\ context specific and is therefore \\ heavily dependent on the point of \\ placement in the care pathways."
}

This editorial is exclusively focused on current challenges faced by multiplexed technologies in low- and middle-income countries (LMICs) in realizing their envisioned implementation.

In LMICs, syndromic management of sexually transmitted and blood-borne infections (STBBI) was the norm for a long time [1]. A lack of affordable screening tests and limited application of evidencebased medicine have continued to fuel this trend. As a consequence, poor quality care became widespread - replete with incomplete treatment and complex care seeking pathways that predispose patients to become lost to follow-up. Recently, a growing awareness about real and perceived harms associated with irrational antibiotic drug prescription for bacterial STIs, coupled with reports of an increase in drug resistance and an increase in funding for diagnostics, have helped catalyze the shift toward the implementation of quality and accurate diagnostics $[2,3]$.

Within many dysfunctional health systems of LMICs, many other factors drive the need to improve the quality of care in at-risk subpopulations. One relevant example is issues of equity and access. On that count, conventional centralized lab systems have slowly observed their limitations in reaching the true populations in need - the marginalized at-risk populations. Such populations often reside in isolated communities, face issues in timely access to care and carry some of the largest burden of undetected coinfections. Yet, many of them remain undiagnosed and untreated. And even when linked to care, it is still challenging to retain them due to their poor socioeconomic status. Additionally, the ever-growing global disease burden of STBBI HIV-associated coinfections adds another layer of complexity. For example, $25-30 \%$ of HIV-infected individuals are coinfected with hepatitis $\mathrm{C}(\mathrm{HCV})$ [4]. In general, the STBBI global burden is an astounding 600 million - approximately 35 million cases of HIV [5], 350 million cases of hepatitis B (HBV) [6], 130 million cases of HCV, 36 million cases of syphilis and 137 million cases of chlamydia and gonorrhea (CT/GC) [7]. Moreover, the control of HIV is contingent on the control of STBBIs in at-risk subpopulations. Their cumulative disease burden poses an economic threat. A cost-effective solution is therefore required in order to

*Faculty of Medicine, McGill University, McIntyre Medical Building, 3655 Sir William Osler, Montreal, Quebec H3G 1Y6, Canada; Nitika.pai@mcgill.ca

\section{KEYWORDS}

- coinfections $\bullet$ HIV $\bullet$ low and middle income $\bullet$ multiplexed $\bullet$ perspective - point of care $\bullet$ sexually transmitted and blood borne infections - technologies 


\section{"Several gaps exist in understanding the full potential of multiplexed POCT. But as data on real world performance and impact measures from ongoing studies emerge, a full evaluation of their risks, benefits and challenges will be informative to policy makers and providers."}

address the growing burden of STBBI and the perceived need to screen populations for several coinfections simultaneously at point-of-care (POC). The offer of simultaneous screening of several STBBIs in close proximity to the patient (within minutes to a couple of hours, of a working day) appears to be promising given its time- saving aspect. This inevitably translates into cost-savings to the healthcare systems, and allows a stress-relief benefit due to the avoidance of another clinic/hospital visit for followup. Additionally, screening for and detecting infections in vulnerable subpopulations - that are continuously at risk for acquisition - could prevent potential future transmission to sexual partners.

With HIV disease burden on a decline, the desire to optimize established HIV platforms to deliver care and treatment for several undetected STBBI seemed to be cost-effective, supposing that it is done efficiently. In this context, an offer of an integrated simultaneous screening, testing, treatment and linkage to care shows potential. These solutions were intended to bring the promise of efficient care to the doorstep of marginalized populations and out of the purview of the care structure of hospitals' central lab systems. Because STBBIs vary in their prevalence/incidence in different at-risk subpopulations, several different permutations and combinations of devices that screen for STBBI exist (HIV/Syphilis, HIV/HCV, HIV/HCV/ HBV, HIV/syphilis/HBV, HIV/CT/GC, HIV/ $\mathrm{CT} / \mathrm{GC} /$ trichomonas/syphilis). All promise an expedited simultaneous conduct, interpretation and execution of rapid POC tests for more than one STBBI (within $30 \mathrm{~min}$ to $2 \mathrm{~h}$ ). The premise is that, a rapid screening solution and linked treatment action plans could potentially impact disease surveillance, detection and control in many subpopulations, thereby averting syndemics. These proposed cost-effective solutions that promise a simultaneous detection of several pathogens (biomarkers or nucleic acid) with integrated POC devices, offer a hope of a rapid and efficient service delivery to healthcare system planners, providers and patients alike. In parallel, an increase in funding from key global agencies has inspired development of many such integrated multiplexed POC technologies [8-10].

Now that many technologies are in development or in evaluation stages, the question remains: will these promised solutions achieve their destiny?
This editorial examines some factors that will predict their impact and sustainability over time.

\section{Type of technology}

Within the multiplexed POC testing (POCT) space, two broad groups of tests and technologies are being developed: single-use biomarkerbased POC tests, and; nucleic acid-based POC platforms.

Biomarker-based multiplexed tests (e.g., Multiplo HBV/HIV/HCV by Medmira [Halifax, Canada]) are primarily antibody- or antigen-based and are used for simultaneous screening/triage of infections with varying accuracies (Sensitivities ranging 95-99\% and specificities ranging $98-99 \%$; there is a risk of false negative/positive results; and dependent on the prevalence of infections). Ideal for mass screening and bio surveillance, these tests are inexpensive, require minimal conditions to operate (no electricity or $\mathrm{Wi}-\mathrm{Fi}$ ) and are feasible to be used in either local resource-challenged labs or centralized-developed labs. However, quality assurance/quality control procedures must be implemented and personnel must be properly trained to reach optimal performance. Moreover, these tests are best used in conjunction with existing robust confirmatory testing algorithms, plans to store and communicate results to the providers and treatment and care pathways.

Platform tests/technologies (e.g., Cepheid's or QIAGEN's) are PCR-based platforms or portable devices that detect nucleic acids [11,12]. Although result turnaround time is slightly longer than an hour with these devices, multiple tests and samples can run concurrently with an accuracy that is claimed to be very high (sensitivity: 99\%, specificity: 99\%) and comparable to lab-based reference standard tests, which is great. They also come with an option to store, relay and retrieve data [13]. Consequently, these devices can be placed in high-throughput settings with massive patient flows (e.g., mobile unit/van-based service delivery points, outreach clinics, etc.). They could potentially serve as diagnostic and confirmatory tests when used in conjunction with patient profile to initiate clinical treatment plans. However, these devices require a certain level of infrastructure (electricity, Wi-Fi and trained personnel) [14] to function optimally and their widespread implementation is limited by their relative high cost and evidence. 


\section{Objectives put to use}

The objectives of multiplexed technologies can vary. These include screening, surveillance, detection, diagnosis, confirmation, linkage to treatment and, when possible, cure. Clarity in stating the objective is not only essential for selecting appropriate technologies but also for optimizing their impact. For example, if the objective is detecting new STBBI cases and linking them to care - to control spread and transmission - then it is imperative to have clinical management protocols in place in settings where they are to be implemented.

\section{Diagnostic performance}

Diagnostic performance determines the success of a technology to a great extent. Interactions at the level of the device, population, patient and systems are important as they can hinder the optimum diagnostic performance of multiplexed POCT. For example, in instances of $\mathrm{HCV} / \mathrm{HIV}$ coinfection, treatment for HIV could reduce infectiousness of $\mathrm{HCV}$, thereby impacting test performance. Sometimes, immunoassay-based devices cannot distinguish between past and recent infection thus impeding rapid clinical action [15]. For this reason, health professionals in endemic settings need to turn to testing algorithms and protocols to decide on the confirmatory test that can resolve the ambiguity and discriminate past from recent infection. However, standardized algorithms for confirmatory testing are not yet in place in many LMICs [16]. Furthermore, in evaluating the performance of platforms, although doubts about their accuracy are uncalled for, confirmation of indeterminate test results may posit a challenge because of expensive algorithms. But procuring confirmatory tests that are superior in accuracy comparatively to the PCR tests will avoid biases in conduct, interpretation and evaluation. This may not be possible in resource-challenged public settings.

\section{Implementation essentials}

Planting a POCT technology is akin to planting a seed in a flowerbed [17]. When the flowerbed is prepared, the probability that a seed will get taken up is high. When implementing a POCT, the intended impact is minimized if much of the assumed infrastructure (flowerbed) is patchy or nonexistent.

In fact, many of the technologies require electricity, Wi-Fi, trained staff, quality assurance/ quality control protocols, confirmatory testing, treatment/linkage care pathways and a buy-in of providers. Many requirements are often not in place in many LMICs.

\section{Impact on costs}

Public health and population level impact of multiplexing needs to be quantified in dollar value using cost-benefit and/or cost-effectiveness analyses. Currently, data on these analyses are sparse. Because there is an implicit difficulty in obtaining estimates on the real burden of STBBIs, quantification of the value proposition can be a challenging exercise in itself. Although it is essential to account for the value that multiplexed technologies bring to the table, it is equally important to assess their market cost. In fact, portable platform technologies that run DNA/RNA tests may have addressed some infrastructure and placement issues, but they may need to reduce their unit cost (which is highly compared with biomarker devices) to allow their placement and global scale up.

\section{Impact on the existing diagnostic, clinical \& public health ecosystem}

There is an established diagnostic and medical ecosystem for the placement of tests and devices. This system, often riddled with corruption, continues to prevail owing to existing business models. The impact of multiplexing is very context specific and is therefore heavily dependent on the point of placement in the care pathways [18]. A new technology that promises efficiency and lower costs may be very valuable to healthcare systems if publicly financed; but in private healthcare systems, these technological solutions are disruptive to the norm. Addressing corruption, continued sales and circulation of inaccurate devices and unsustainable retail markups, are central to the scale up and sustainability of multiplexed POC tests. Yet, these issues remain untapped for fear of repercussions.

\section{Conclusion}

In 2015, the diagnostic landscape of multiplexed technologies appears to be very promising. Yet, infrastructural issues and unresolved questions about their impact and incorporation within existing business models may not allow a rapid and efficient uptake. Integrated linkages to treatment will also depend on the settings. Technologies are to be used within health systems and so their implementation cannot
"Unless current policies are changed, their envisioned scale up will not be grounded on data driven evidence. Until then, their introduction has to be therefore guided by a healthy dose of caution and prudence, and a lack of both can hinder their optimum placement, thereby impacting their sustainability over time." 
ignore this obvious fact. Several gaps exist in understanding the full potential of multiplexed POCT. But as data on real world performance and impact measures from ongoing studies emerge, a full evaluation of their risks, benefits and challenges will be informative to policy makers and providers. These data will generate an adequate level of buy-in among key stakeholders. These key professionals are currently sold on the concept but need solid scientific- and evidence-based data to make informed decisions on multiplexed technologies for STBBIs. Unless current policies are changed, their envisioned scale up will not be grounded on data driven evidence. Until then, their introduction has to be therefore guided by a healthy dose of caution and prudence, and a lack of both can hinder their optimum placement, thereby impacting their sustainability over time.

\section{Acknowledgements}

The author thanks J Daher for her valuable feedback.

\section{Financial \& competing interests disclosure}

Operating grant has been received from Grand Challenges Canada's Stars in Global Health. N Pant Pai acknowledges the support of operating grants from the CIHR, Gates Foundation and a salary award Junior 1 from the FRSQ. The author has no other relevant affiliations or financial involvement with any organization or entity with a financial interest in or financial conflict with the subject matter or materials discussed in the manuscript apart from those disclosed.

No writing assistance was utilized in the production of this manuscript.

\section{References}

1 World Health Organization. Guidelines for the Management of Sexually Transmitted Infections. WHO, Geneva, Switzerland (2001).

2 Lewis DA, Lukehart SA. Antimicrobial resistance in Neisseria gonorrhoeae and Treponema pallidum: evolution, therapeutic challenges and the need to strengthen global surveillance. Sex Transm Infect. 87(2), ii39-ii43 (2011).

3 World Health Organization. Emergence of multi-drug resistant Neisseria gonorrhoeaethreat of global rise in untreatable sexually transmitted infections. WHO, Geneva, Switzerland (2011).

4 Alter MJ. Epidemiology of viral hepatitis and HIV co-infection. J. Hepatol. 44(1 Suppl.), S6-S9 (2006).

5 Joint United Nations Programme on HIV and AIDS. Global Report: UNAIDS report on the global AIDS epidemic 2013. WHO, Geneva, Switzerland (2013).
6 World Health Organization. Hepatitis B. (2002).

http://www.who.int

7 World Health Organization. Global incidence and prevalence of selected curable sexually transmitted infections 2008. WHO, Geneva, Switzerland (2012).

8 Qualcomm Tricorder. Overview. http://tricorder.xprize.org/about/overview

9 GenomeWeb. Gates Foundation Awards $\$ 100 K$ to Lab-on-a-Chip Non-profit Diagnostics for All. https://www.genomeweb.com

10 Grand Challenges Canada. Fabric Chips: a versatile platform for low-cost, rapid and multiplexed diagnostics. http://www.grandchallenges.ca

11 Cephed. Cepheid RemoteXpert Platform. http://manas.com

12 QIAGEN. Prevention. https://www.qiagen.com

13 Cepheid. Corporate overview. http://www.google.ca
14 Denkinger CM, Grenier J, Stratis AK, Akkihal A, Pant-Pai N, Pai M. Mobile health to improve tuberculosis care and control: a call worth making. Int. J. Tuberc. Lung Dis. 17(6), 719-727 (2013).

15 Pai NP, Dhurat R, Potter M et al. Will a quadruple multiplexed point-of-care screening strategy for HIV-related co-infections be feasible and impact detection of new co-infections in at-risk populations? Results from cross-sectional studies. BMJ Open. 4(12), e005040 (2014).

16 Pant Pai N, Daher J. Multiplexed testing for HIV and related bacterial and viral co-infections at the point-of-care: quo vadis? Expert Rev. Mol. Diagn. 15(4), 463-469 (2015).

17 Pant Pai N, Behlim T. Point-of-care technologies and their global health applications. Curr. Pharmacogenomics Person. Med. 11(3), (2013).

18 Pai M, Ghiasi M, Pant PN. Point-of-care diagnostic testing in global health: what is the point? Microbe 10 (3), 103-107 (2015). 